\title{
Conservative solutions to the black hole information problem
}

\author{
Sabine Hossenfelder and Lee Smolin \\ Perimeter Institute for Theoretical Physics \\ Waterloo, Canada N2J $2 W 9$ \\ and \\ Department of Physics, University of Waterloo
}

October 22, 2018

\begin{abstract}
We review the different options for resolution of the black hole loss of information problem. We classify them first into radical options, which require a quantum theory of gravity which has large deviations from semi-classical physics on macroscopic scales, such as non-locality or endowing horizons with special properties not seen in the semi-classical approximation, and conservative options, which do not need such help. Among the conservative options, we argue that restoring unitary evolution relies on elimination of singularities. We argue that this should hold also in the AdS/CFT correspondence.
\end{abstract}

\section{Contents}

1 Introduction

2 Definitions: asymptotic structures for quantum spacetimes

3 The options for the fate of quantum black holes

Option 1: Hawking's scenario: there are both horizons and singularities . . . . . 8

Option 2: Quantum naked singularities . . . . . . . . . . . . . . 9

Option 3: Neither horizon nor singularity . . . . . . . . . . . . . . . . . . 9

Option 4: There is an horizon but no singularity . . . . . . . . . . . . 11

The cost of saving quantum mechanics . . . . . . . . . . . . . . . . . 14

4 Other issues $\quad \lcm{14}$

4.1 Objections against stable, or quasi-stable remnants . . . . . . . . . . . 14

$4.2 \mathrm{AdS} / \mathrm{CFT} \ldots \ldots \ldots \ldots \ldots \ldots \ldots \ldots$

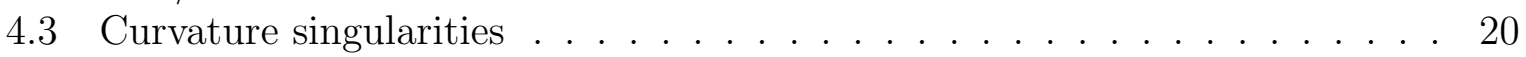

5 Discussion $\quad 21$

6 Conclusions $\quad \lcm{22}$ 


\section{Introduction}

The conjecture that information is lost, and unitarity fails, when black holes evaporate, has challenged theorists since Hawking first proposed it in 1974 [1, 2]. After more than three decades of discussion, there have been many proposals, but no consensus on a solution. In this comment, we would like to contribute to the solution of the problem by applying some basic logic to the different options on the table. We will argue that by making two simple classifications of the possible solutions one can argue, even in the absence of a complete quantum theory of gravity, what the plausible resolution is.

The first classification has to do with how heroic or radical are the steps asked of the quantum theory of gravity. We will label a proposed solution to the information loss puzzle radical if

- It attributes to the horizon or apparent horizon of a black hole physical properties which are not also properties of arbitrary null surfaces, or which are not apparent in a semiclassical treatment.

- It calls on extreme forms of non-locality in regions of spacetime with curvatures far below the Planck scale, or requires transfer of quantum information over large spacelike intervals.

If either or both of these things are true then the quantum theory of gravity attribute to weakly curved regions of space-time properties very different from those found in the semiclassical description. Any approach to resolution of the black hole information puzzle that does not make either of these assumptions will be called conservative.

There have been claims made that string theory has mechanisms that can underlie a radical solution of the problem [3], though the relevance of these effects has been debated in [4]. We think it is fair to say that the questions of whether or not string theory requires or admits a radical solution to the information loss problem are presently open.

We would then like to advocate the view that before trying to make the radical solutions work we should make sure that an acceptable resolution does not come out of a conservative approach. To begin with, it is not terribly plausible that the horizon would be the source of the resolution to the problem, because in classical and semi-classical General Relativity, the horizon is locally no different from any other null surface. It is only what happens globally, that determines if a surface is an horizon or not. Because of this, it seems very unlikely that a quantum theory of gravity will do much to modify the physics near horizons. At most one can expect a little bit of quantum fluctuations present at the horizon to induce a quantum ergosphere, as discussed by York in [5]. But this is not enough, by itself, to solve the black hole information puzzle.

But if we don't have reason to expect much from the horizon, the one place we do expect a quantum theory of gravity to have a major impact is on the singularity and the region approaching it, where the curvature invariants exceed Planck scales. In fact, the idea that quantum gravity will remove the spacelike singularities of black holes and cosmological models is very old and supported by calculations in a variety of models and approaches [6, 7, 8]. 
In this paper we will not consider the question of whether particular approaches to quantum gravity resolve singularities. We will, instead, assume that some theory does remove singularities and ask whether by doing so it leads to a resolution of the black hole information puzzle. But we do note that there is recent work that does show that, in a particular model of quantum gravity, black hole singularities are removed in a way that leads to the restoration of unitary evolution. This result has been derived in a study of the CGHS [9] model by Ashtekar et al in [10]. They find results that confirm earlier arguments in [12]. Part of the motivation of this paper is to put their results in a broader context.

Let us consider the evolution, within a quantum theory of gravity, of the spacetime of a star on the verge of collapse to a black hole. We can call the result a quantum black hole spacetime. This kind of initial condition can be specified entirely within a semi-classical region. We can be sure that such a spacetime will have a region of trapped surfaces, and hence an apparent horizon. In such a context we can ask two questions, the answers to which lead to a classification of the conservative options for a resolution of Hawking's puzzle:

1. Is there a real event horizon?

2. Is there a singularity?

Each of these has two possible answers, yes and no.

We will first need to give more precise definitions of the terms involved. Once this is done we may consider the four possibilities that come from the possible answers to these two questions. We will see that with minimal assumptions about the quantum theory of gravity, and more precise definitions of the terms involved, we can reach a simple and robust conclusion:

- Unitarity restoration in a conservative context requires a quantum theory of gravity that eliminates singularities.

We will also argue below, that in a quantum theory of gravity where probability is well defined, and therefore conserved, the absence of singularities plausibly implies there is unitary evolution. This then complements the results of Ashtekar et al [10] which shows that in a specific model the singularity is eliminated and unitary evolution is restored.

In this paper we strive to make assumptions about the quantum theory of gravity only to the extent they are needed to frame the initial paradox of Hawking and a resolution of it. Thus, while we will give criteria for the presence of horizons and the absence of singularities, we make these the weakest criteria needed to draw definite conclusions. As such, we expect our conclusions to hold in a large variety of theories and contexts. We do not aim to provide a concise review of the literature or previously discussed solutions. Nor do we claim the point of view advocated here is original, our aim is to support an approach to resolving the problem that seems to have been underappreciated 1 . For reviews on the black hole information loss problem the interested reader is referred to [14, 15].

\footnotetext{
${ }^{1}$ Other authors who proposed conservative solutions include [13, 37.
} 
In the next section we give some essential definitions, which makes it possible to classify the different options for a conservative resolution of the black hole information problem in section 3. In section 4 we discuss a few other issues, including objections to remnants and baby universes and the extension of our arguments to the AdS/CFT context. $m_{\mathrm{Pl}}$ and $l_{\mathrm{Pl}}$ denote the Planck mass and Planck length respectively.

\section{Definitions: asymptotic structures for quantum space- times}

We will start with defining some necessary terminology. Our aim is to make statements about a space-time with an initially semi-classical state whose evolution however might have a quantum gravitational phase or region. We will not define what a quantum spacetime is, as that depends on the approach to quantum gravity, about which we do not want to make any specific assumptions. We are interested only in a definition of quantum spacetimes which are semi-classical in the following sense:

A quantum spacetime $\mathcal{Q S} \mathcal{T}$ is partly semi-classical if

a) There is a procedure to define a manifold $\mathcal{R}$, a metric operator $\hat{g}_{a b}$ and a state $|\Psi\rangle$, such that $\left(\mathcal{R},\left\langle\Psi\left|\hat{g}_{a b}\right| \Psi\right\rangle\right)$ defines a possibly extendible Lorentzian spacetime (which we call for short $\left.\left(\mathcal{R},\left\langle g_{a b}\right\rangle\right)\right)$.

b) The semi-classical Einstein equations for $\left\langle g_{a b}\right\rangle$ are satisfied on $\mathcal{R}$, up to small quantum corrections.

Here, $\left(\mathcal{R},\left\langle g_{a b}\right\rangle\right)$ may not be complete, ie there may be regions of $\mathcal{Q S T}$ that do not have a classical or semi-classical description. If the quantum theory of gravity still allows us to define a manifold $\mathcal{M}$ such that $\mathcal{R} \subset \mathcal{M}$, then the $\mathcal{X}=\mathcal{M}-\mathcal{R}$ constitutes the quantum region of the quantum spacetime, while $\mathcal{R}$ constitutes its semi-classical region and $\left(\mathcal{R},\left\langle g_{a b}\right\rangle\right)$ is its classical approximation.

Since the $\mathcal{Q S T}$ could have several semi-classical regions, we further need

The classical approximation of a quantum spacetime is semi-classically complete if it contains all the regions of spacetimes that can be defined by the procedure that defines $\left(\mathcal{R},\left\langle g_{a b}\right\rangle\right)$.

We can then make three important definitions

A partly semi-classical quantum spacetime $\mathcal{Q S T}$ is asymptotically flat if its classical approximation $\left(\mathcal{R},\left\langle g_{a b}\right\rangle\right)$ is asymptotically flat.

Depending on the quantum theory of gravity, a quantum spacetime $\mathcal{Q S T}$ may or may not have a causal structure that extends that of its classical approximation. If it does not then the causal structure of a quantum spacetime is the causal structure of its classical approximation, otherwise the latter is a sub partial order of the former. In either case, we can make several key definitions. 
A region of a quantum spacetime $\mathcal{Q S T}$ that is not in the causal past of $\mathcal{I}^{+}$of its classical approximation $\left(\mathcal{R},\left\langle g_{a b}\right\rangle\right)$ is an asymptotically future hidden region.

We can then say

A quantum spacetime $\mathcal{Q S T}$ has a future event horizon if it contains an asymptotically future hidden region.

Note that we did not define the horizon itself, but merely a condition for its presence. We do this since we can not make statements about the quantum gravitational regions. These definitions are well defined whether or not there is a quantum causal structure that extends the causal structure of its classical approximation. The same is true of the following.

A complete spacelike surface $\Sigma$ of a quantum spacetime is a set of events of which no two are time- or lightlike to each other, and to which no events can be added without violating this condition.

If the causal structure does not extend past the classical approximation, then a spacelike surface is contained within the semi-classical region, otherwise it may extend.

Given such a complete spacelike surface we can linearize the quantum gravity theory (together with any matter fields) in its neighborhood and define a quantum theory of small fluctuations around $\Sigma$. We will assume that associated to any complete spacelike surface $\Sigma$ of a quantum spacetime there is an operator algebra $\mathcal{A}_{\Sigma}$ of local fields that describe small fluctuations in fields around the state $|\Psi\rangle$, which has a representation in a Hilbert space $\mathcal{H}_{\Sigma}$.

We do not necessarily know what a singularity of a quantum spacetime is, but we can define a condition equivalent to the absence of any singularities:

The quantum spacetime is quantum non-singular if for any two complete non-intersecting spacelike hypersurfaces $\Sigma_{1}$ and $\Sigma_{2}$, there is a reversible linear map $\mathcal{M}_{1,2}: \mathcal{H}_{1} \rightarrow \mathcal{H}_{2}$, corresponding to dynamical evolution. Otherwise we say that the quantum spacetime is quantum singular.

Note that any slice means any slice in any slicing. The motivation for this definition is the following. The spacelike singularities in black holes that are the source of information loss in classical and semi-classical General Relativity do so by disabling the possibility for time reversal - specifically time reversal in the theory of small fluctuations propagating on a background spacetime. If spacetime has a classical singularity it is geodesically incomplete [16], meaning there exists at least one time- or lightlike geodesics that can not be continued through the singularity. For this geodesic that ends at the singularity in a final point, consider a complete spacelike slice that meets the singularity in the same point. Whatever the information of the particle was that hit the singularity on this incomplete 
geodesic, it does not get passed on to the spacelike slice 2 . The above definition is a generalization of this classical definition without the explicit referral to geodesics, since it is a notion that might not be well-defined in the quantum gravitational region.

One way to think about the loss of information in black hole evaporation is that less information is present after the evaporation in Hawking's original scenario because some of the information from $\mathcal{I}^{-}$goes into the singularity and does not get out to $\mathcal{I}^{+}$. But looking at the time reversed situation this is not the case, all the information near $\mathcal{I}^{+}$ can be obtained from $\mathcal{I}^{-}$. Now $\mathcal{I}^{ \pm}$are not spacelike hypersurfaces but they represent the fact that in either slicing one will eventually (potentially at $t \rightarrow \pm \infty$ ) have to face the presence of the singularity. We could then propose the slightly different criteria for non-singularity that there is a reversible map between the Hilbert spaces for incoming and outgoing modes of a massless fields constructed on $\mathcal{I}^{+}$and $\mathcal{I}^{-}$. The definition we have given implies this in the case of asymptotically flat spacetimes, because, in the absence of a classical singularity, $\mathcal{I}^{+}$and $\mathcal{I}^{-}$are each the limits of complete spacelike surfaces. But the definition we have given is more general and, as we will see, applicable to the case of asymptotically AdS spacetimes.

So we propose that a sufficient indication that no singularity is present is that the time evolution can be reversed: if one can propagate information forward from $\Sigma_{1}$ to $\Sigma_{2}$, one can propagate it backwards from $\Sigma_{2}$ back to $\Sigma_{1}$.

Note that we do not require either that some curvature invariants blow up or some condition of geodesic incompleteness. What is needed is only that the propagation of the field modes between any two such surfaces can be used to define a reversible transformation between the Hilbert spaces associated with those surfaces. This is good because we do not know if a particular quantum theory of gravity will allow us to define either curvature or geodesics in the quantum region. But we expect that if there is a singularity, in either of those senses, the condition we have just given of non-singularity can not be satisfied. Since the latter can be defined more generally it is appropriate our purposes. We will discuss the relation to curvature singularities in section 4.3 .

Now reversibility is we may note, weaker than unitarity; it is a necessary, but not a sufficient requirement for unitarity. But we can argue that if the theory of small fluctuations propagating on the quantum spacetime has a sensible definition of quantum probability, then the map $\mathcal{M}_{1,2}$ will also be unitary. An heuristic argument for this goes as follows. If necessary, impose infrared and ultraviolet cutoffs so that the Hilbert spaces $\mathcal{H}_{1}$ and $\mathcal{H}_{2}$ are finite dimensional. Since there is an invertible map between them they must have the same dimension, $n$. Then the linear map $\mathcal{M}_{1,2}$ is an element of $G L(n, C)$ with nonvanishing determinant. If we however impose the plausible requirement that probability as determined by the inner products, is preserved in any basis, then $\mathcal{M}_{1,2}$ is an element of $U(n)$.

This can be seen in the well studied example of particle production in time-dependent backgrounds, reviewed in [17, 18]. Consider that the fluctuations in question are described

\footnotetext{
${ }^{2}$ If spacetime is time-or lightlike geodesically incomplete it is in principle possible the spacelike slice can be continued through the singularity. This does not affect the argument since it is only relevant the geodesic can not be continued.
} 


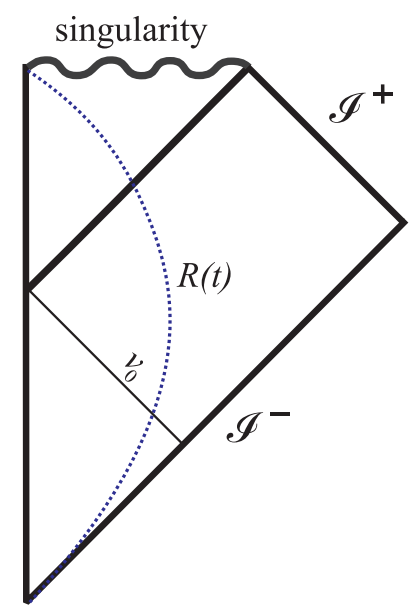

Figure 1: Conformal Penrose diagram for the formation of a classical black hole. The dotted (blue) line indicates the surface of the collapsing matter with radius $R(t)$. The solid (black) line is the event horizon, the thin (black) line labeled with $\nu_{0}$ is the last ray of light that escapes the fate of falling into the singularity.

by a scalar field $\hat{\phi}$ satisfying the usual massless scalar field equation, $\nabla_{a} \nabla^{a} \hat{\phi}=0$ in the semi-classical regions. Assume also that there is a time coordinate, $t_{1}$, in the neighborhood of $\Sigma_{1}$, such that $\Sigma_{1}$ is defined by $t_{1}=$ constant, and similarly for $\Sigma_{2}$. Then we can follow the usual construction in which field operators are decomposed into creation and annihilation operators based on positive and negative frequencies defined by $t_{1}$ and $t_{2}$, and construct the associated Fock spaces. We assume that the modes of $\hat{\phi}$ propagate between any two spacelike surfaces, whether semi-classical or not, so that, $\mathcal{M}_{1,2}$ is a Bogoliubov transformation between the creation and annihilation operators in $\mathcal{A}_{\Sigma_{1}}$ and $\mathcal{A}_{\Sigma_{2}}$. Given the completeness of the two surfaces, if there are no singularities where information of the modes might end up, then a unitary transformation between $\mathcal{H}_{\Sigma_{1}}$ and $\mathcal{H}_{\Sigma_{2}}$ can be constructed from the Bogoliubov transformation [19]. On the other hand, if one considers an incomplete slice as final region, the evolution will generically be non-unitary and evolve pure into mixed states.

The final defintion we need is that of a quantum black hole spacetime. The following will be sufficient for our purposes.

A partially semi-classical, asymptotically flat quantum spacetime $\mathcal{Q S T}$ is a quantum black hole spacetime if it has a complete spacelike slice in the semi-classical region with initial conditions that would classically lead to the formation of a horizon and a black hole.

Assuming the standard positive energy conditions, were there no quantum effects the formation of an horizon and a singularity, in the senses we have defined here, would be inevitable and result in the classical case of black hole formation as depicted in Figure 1. The question, to which we now turn, is what can happen in the quantum case.

We will in the following restrict our attention to non-rotating, uncharged black holes. 


\section{The options for the fate of quantum black holes}

For the discussion of this paper we will assume the existence of a quantum theory of gravity for which the definitions we have just given make sense. There are four logically possible scenarios to the evolution, which we now describe.

\section{Option 1: Hawking's scenario: there are both horizons and sin- gularities}

Hawking's original argument [1, 20] for a loss of information uses the following setting:

Hawking's original scenario: Let $\mathcal{Q S T}$ be a quantum black hole spacetime. Then it will have an horizon and will be singular, according to the definitions above.

We can refer to Figure 2 for a view of what he had in mind. In this case we say that unitary evolution has broken down and there is loss of information because there are two surfaces $\Sigma_{1}$ and $\Sigma_{2}$ such that there is no reversible map from $\mathcal{H}_{1}$ to $\mathcal{H}_{2}$. No matter what the initial state was, the outcome is always a thermal state and it is not possible to find out from the final state what the initial state was.

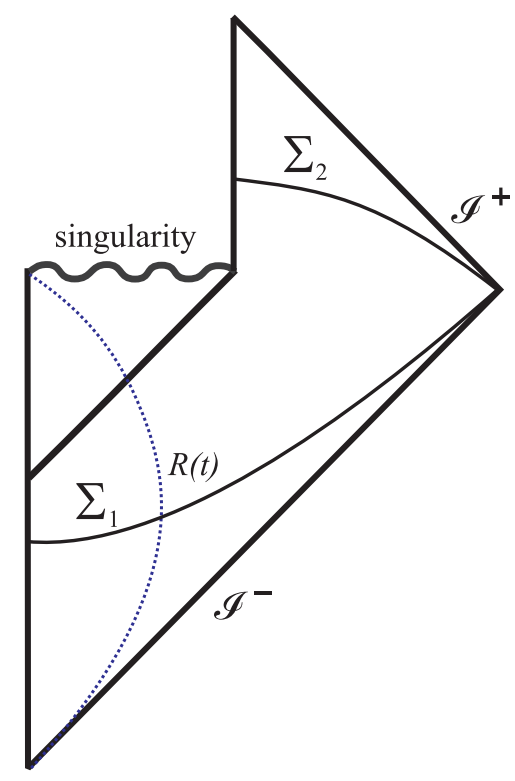

Figure 2: Hawking's evaporation scenario. The dotted (blue) line indicates the surface of the collapsing matter with radius $R(t)$. The solid (black) line is the event horizon, the thin (black) lines labeled with $\Sigma_{1 / 2}$ are two complete spacelike hypersurfaces.

Note that in Hawking's original argument the quantum theory of gravity retained the singularity of the classical and semi-classical black hole. As we will see shortly, one obtains a potential paradox only when this assumption is made. We can conclude that 
Hawking was correct, in arguing that if the black hole spacetime retains an event horizon and spacelike singularity in the full quantum theory, then information is lost and unitarity is impossible.

But let us see what the other options are.

\section{Option 2: Quantum naked singularities}

Quantum naked singularity scenario: Let $\mathcal{Q S T}$ be a quantum black hole spacetime. Then in the quantum theory it will be singular but have no event horizon.

This is the worst possible outcome and, to our knowledge, no one is proposing it. We mention it for completeness. It would mean that whatever happens in the quantum region is not unitarily determinable by the past but is not hidden behind an horizon and can affect the whole spacetime out to $\mathcal{I}^{+}$.

Let us now discuss two ways that the information paradox could be resolved by a quantum theory.

\section{Option 3: Neither horizon nor singularity}

Complete evaporation scenario: Let $\mathcal{Q S T}$ be a quantum black hole spacetime. Then in the quantum theory of gravity it will nonetheless be nonsingular and it will be without an event horizon.

This is illustrated by Figure 3, In spite of there being an apparent horizon, indicated by the thick dotted line, there is not a real event horizon. The apparent horizon ends at point E, after which we can say that the black hole has evaporated. Plausibly, this would occur when the black hole has shrunk down to Planckian size. Since the trapped surface could vanish in this quantum gravitational phase, it might not be useful to think of this object as a black hole anymore. In this scenario, all the information that was trapped within the apparent horizon could eventually get out to infinity. Since the quantum spacetime is non-singular it follows that any complete spacelike surface $\Sigma_{2}$ to the future of $\mathrm{E}$ has a state which is the image of a map from the pure state in $\mathcal{H}_{1}$ on the initial value surface $\Sigma_{1}$. The initial state thus can be recovered from the final one and there is no obstacle to there being a unitary map from a state on an initial surface to a state on a final surface.

This kind of scenario was discussed in [47, 11], was later advocated by Ashtekar and Bojowald [12] and a detailed example of this kind of resolution was then provided by the treatment of the CGHS model [9] by Ashtekar et al in [10]. This is a $1+1$ dimensional model of quantum gravity coupled to a scalar field, motivated by string theory [21, 22]. The classical model has black hole solutions, and semi-classical calculations show that there is Hawking radiation and hence an information loss puzzle. Ashtekar et al show in two controlled approximations that the singularity and horizon are eliminated in the 


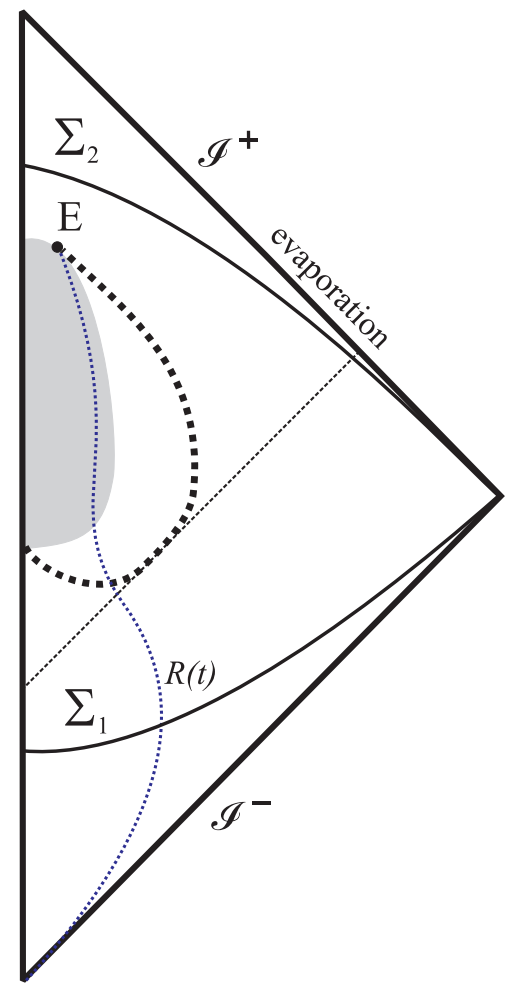

Figure 3: Option 3: Complete evaporation scenario. The grey shaded region is potentially subject to non-negligible quantum gravitational corrections. The thick dotted line is the apparent horizon, the thin dotted (blue) line indicates a possible world line of the surface of the collapsing matter configuration. The thin dashed line represents the lightlike surface where the event horizon of the collapsing matter had been without evaporation.

quantum theory, leading to unitary evolution and a resolution of the information loss puzzle.

One key aspect of the Ashtekar et al work is that their methods allow them to study quantum spacetimes that have both regions which are semi-classical, AND regions which are far from classical, characterized by very large fluctuations in expectation values of operators that measure the metric geometry. The existence of quantum spacetimes which are in this way, non-uniformally approximated by semi-classical or effective field theory methods is crucial to a resolution of the puzzle of black hole evaporation. This is because without regions which are semi-classical, which include asymptotic regions with timelike killing fields, the problems of unitarity and information loss can not be posed precisely enough to investigate. That is, unless regions far from the black hole can be treated semi-classically, the context in which Hawking's puzzle arises can not be reproduced. At the same time, the elimination of the singularity requires a region of space-time where the semi-classical approximation fails badly. Arguments against this scenario have been offered on the basis of a claim that it would lead to long lived remnants. We will discuss these in section 4 


\section{Option 4: There is an horizon but no singularity}

A second way that quantum gravity could resolve the conjecture without loss of information is in the following scenario.

Massive remnant or baby universe scenarios: Let $\mathcal{Q S T}$ be a quantum black hole spacetime. Then in the quantum theory of gravity it will be non-singular, but there will still be an horizon.

There are then two possibilities of such remnants:

4A: To the future of the event horizon, all complete spacelike hypersurfaces decompose into two regions that are not connected by any spacelike curves. There is then a region that pinches off from the asymptotically flat region, forming a new region of spacetime that we will refer to as a baby universe.

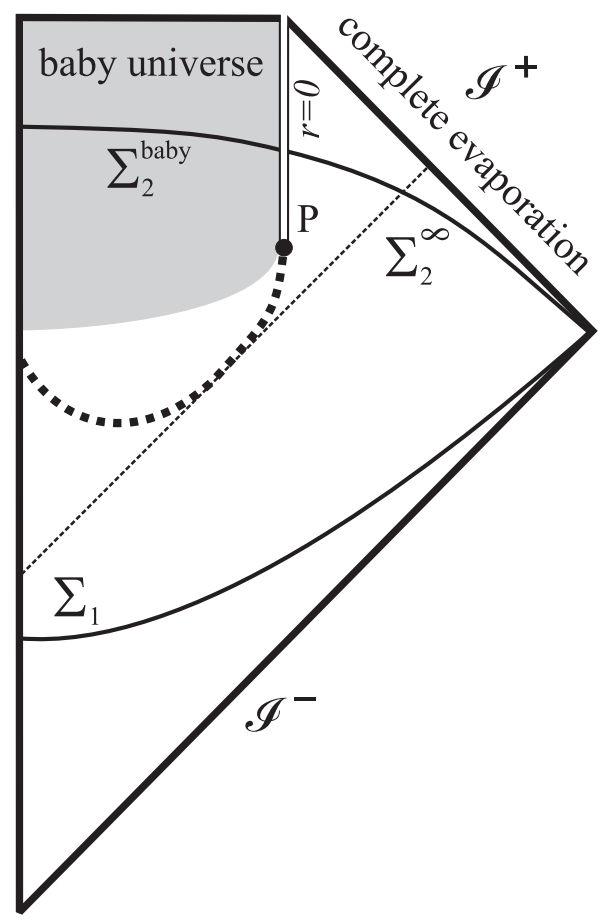

Figure 4: Option 4A: Example of a baby universe scenario. The pinch-off point is marked with $\mathrm{P}$, the thick dotted line indicates the apparent horizon. The double thin line is a boundary between two disconnected regions. The grey shaded region is potentially subject to non-negligible quantum gravitational corrections. The thin dashed line represents the lightlike surface where the event horizon of the collapsing matter had been without evaporation.

An example for this case is illustrated in Figure 4. The pinch off point is event P. There the 2-surfaces making up the apparent horizon shrink down to zero area, but the volume 
contained within remains finite. The resulting pinch off is illustrated in Figure 4. In this case there is no quantum singularity because there are complete surfaces, $\Sigma_{2}=\Sigma_{2}^{\infty} \cup \Sigma_{2}^{\text {baby }}$ to the future of $\mathrm{P}$ which have two components, a component with an asymptotic boundary at spatial infinity and a compact component within the baby universe. There is no loss of information and no obstacle to unitary evolution, because the quantum information that falls behind the horizon survives indefinitely in the baby universe. So the whole universe at a later time has a pure state, even if the component connected to $\mathcal{I}^{+}$can only be described by a density matrix. This kind of resolution of the problem has been proposed by [6, 7, 31, 37].

4B: The horizon never shrinks down to zero size but stabilizes at a finite radius, forming a permanent massive remnant, surrounded by an event horizon. In contrast to scenario $4 \mathrm{~A}$, the spacelike surfaces do not fall apart into two disconnected regions, and the remnant never disappears from the original spacetime.

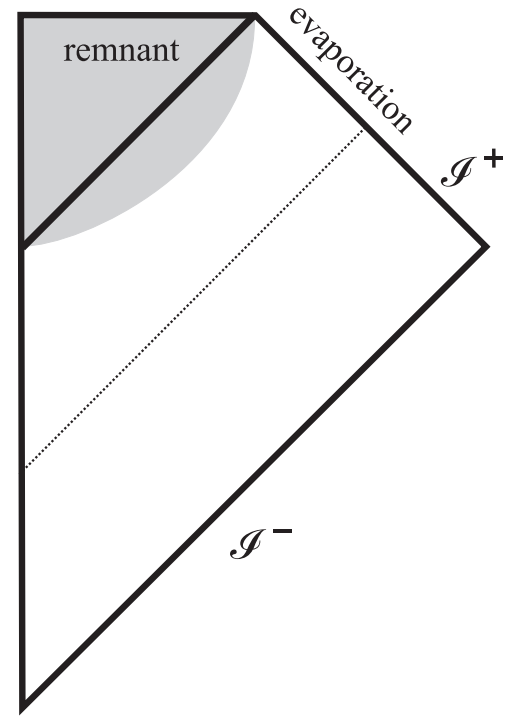

Figure 5: Option 4B: Example for a massive remnant scenario. The solid line depicts the event horizon. The thin dashed line represents the lightlike surface where the event horizon of the collapsing matter had been without evaporation. The grey shaded region is potentially subject to non-negligible quantum gravitational corrections.

In this case, unitary evolution can be maintained when the degrees of freedom in the interior of the remnant are taken into account. This is not that different in consequences from the baby universe scenario, as the spatial volume of the interior of the remnant may grow arbitrarily large (in comparison to $A^{\frac{3}{2}}$, where $A$ is the minimal area of the throat connecting the original spacetime to the remnant ${ }^{3}$ ) while the exterior remains an horizon

\footnotetext{
${ }^{3}$ Spherical symmetry allows an unambiguous definition of the three-volume [45].
} 
of fixed size. In contrast to option 4A, the massive remnant's ADM mass and its surface never reach zero but they stabilize at a finite value. In either case unitary evolution is preserved, but there is a real horizon because the information trapped in the baby universe or remnant can never reach $\mathcal{I}^{+}$. This case is depicted in Figure 5. In both cases, $4 \mathrm{~A}$ and $4 \mathrm{~B}$, the evolution is unitary on complete hypersurfaces but will seem non-unitary in the exterior due to the incompleteness of the final region

There are some objections to these scenarios which we will discuss below.

For completeness we note two variants of this option. To the future of the disconnected interior, there might follow another spacelike non-compact region that can be asymptotically flat, an example is shown in Figure 6, left. Or, if the disconnected region reconnects to the mother universe, we have an alteration of option 3, depicted in Figure 6, right. We emphasize however that though possible, both of these scenarios are unlikely in a conservative approach, because they are not evolutions of complete initial data on $\mathcal{I}^{-}$. In the first case $\mathcal{I}^{-}$is not the complete initial data surface, in the second there is no dynamics known that could mediate the reconnection of disjoint regions of quantum spacetime.
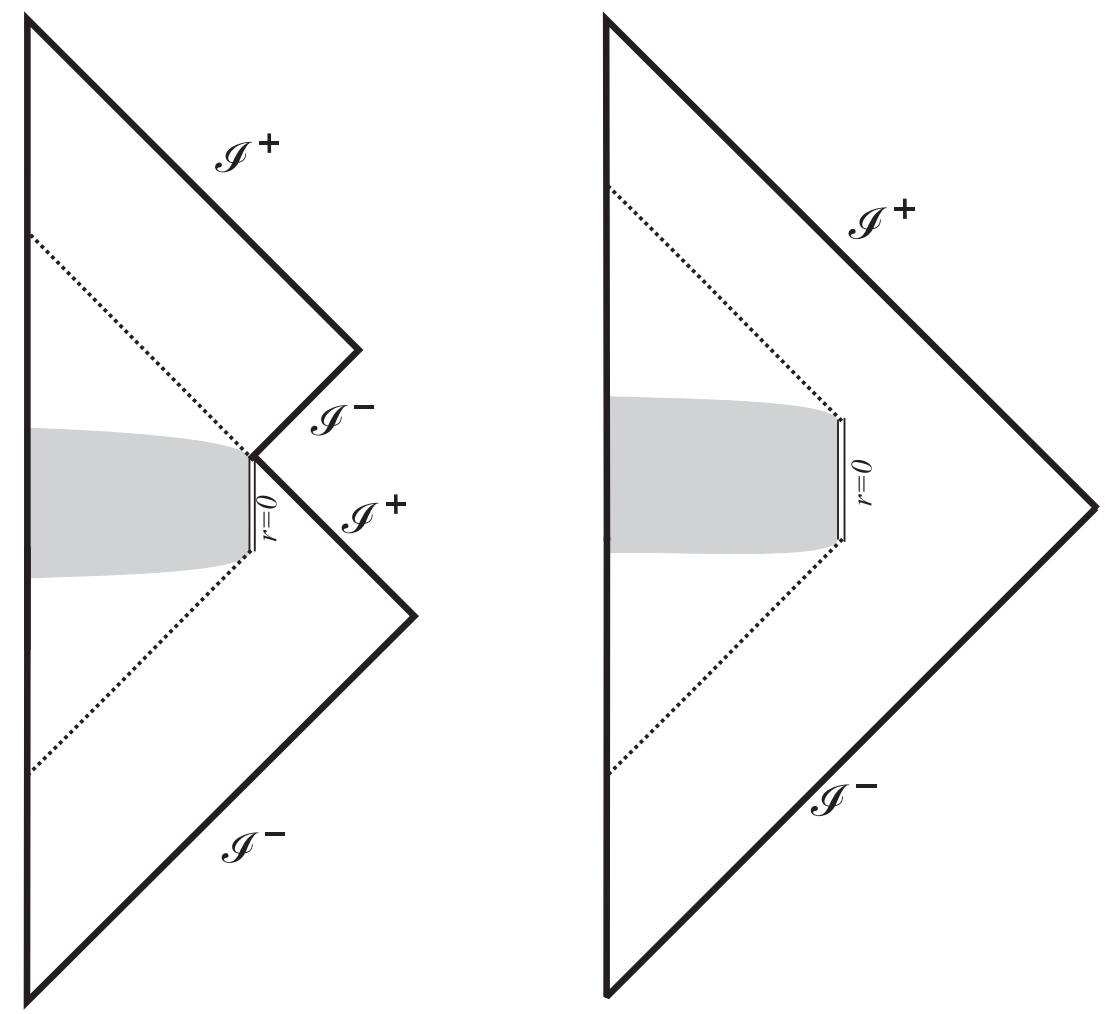

Figure 6: Left: Option 4A: Example for baby universe scenario with a second asymptotically flat region. Right: Option 3: Example for an intermediate disconnected region that later reconnects. The double thin lines indicates a boundary between two disconnected regions. Grey shaded regions are potentially subject to non-negligible quantum gravitational corrections. 
In all of the above cases, we can not tell for certain how far the quantum gravitational regions will extend and whether an intermediate quantum region might be followed by another semi-classical region. The grey shaded regions in all of these figures indicate the possible presence of quantum gravitational effects, though they might indeed be negligible in some of these areas. For example, if the interior of the baby universe grows very large, one would expect that quantum gravitational effects only play a role at the junction of the mother- and baby universe to resolve the singularity.

\section{The cost of saving quantum mechanics}

These four cases cover the possible options for a conservative resolution of the black hole information problem, ie those in which the quantum black hole spacetime has or has not an horizon or singularity. The first and second inevitably suffer from a loss of information, since the evolution is not time reversible, which means in particular the evolution of quantum states cannot be unitary. The third and fourth have reversible evolution. Reversibility is necessary, though not sufficient, for unitary evolution, but as we have argued earlier, a theory that has reversible but non-unitary evolution would lack a sensible definition of quantum probability.

The third and fourth scenario eliminate the classical singularity and so both have quantum information existing to the future of where the classical singularity would have been. They differ only on whether an observer near $\mathcal{I}^{+}$could recover that information. If she can then there was not a real horizon, only an apparent one. If she can't then the information still exists, but is somewhere she can never see it, either in a permanent remnant $(4 \mathrm{~B})$ or in a new region of spacetime that has pinched off from hers and from now on evolves on its own $(4 \mathrm{~A})$.

Thus, the cost of saving quantum mechanics, without reliance on radical modifications of spacetime in the semi-classical domain, is either a baby universes, a remnant, or a quantum region that eventually leaks out its quantum information to infinity.

If one wants or hopes that quantum theory is not be modified fundamentally by its incorporation of gravity, so that the resolution of the black hole information problem is conservative, then one wants one of these scenarios to work out. We see that the important thing to understand, to resolve the problem, is what happens in the quantum gravitational phase and how the singularity is avoided in the quantum theory of gravity.

\section{Other issues}

\subsection{Objections against stable, or quasi-stable remnants}

There is a literature critical of the option where there remain remnants, either permanent or long lived [23, 24, 25]. If these are correct there remain two conservative options: a baby universe or a quick decay of the quantum region without a long lived remnant. Nonetheless, the arguments against remnants are not, in our view, definitive, for reasons we would like to explain here. 


\section{There is not enough information remaining inside a remnant}

There is a weak and a strong interpretation of the Bekenstein-Hawking entropy [26, 27, 28, 29. The weak form says that the black hole entropy $S_{\mathrm{BH}}=M^{2}$ (in Planck units) is a measure of the number of bits of quantum information that could be gotten by measurements made at the horizon. Another way to say this is that the Bekenstein bound is a measure of channel capacity for an apparent horizon. The strong form asserts that $S_{\mathrm{BH}}$ is the amount of quantum information needed to specify the state to the interior of the horizon, ie log of the dimension of the subspace of Hilbert space needed to describe the interior. There are several reasons for ruling out the strong form, which are reviewed in [27].

For example, if one takes the strong form one then must consider the following problem. A black hole formed in the past at mass $M_{\mathrm{i}}$ and then evaporated down to the present when its mass is $M_{\mathrm{f}}$. The semi-classical calculations asserts that the black hole has produced thermal radiation that does not carry any information (except its temperature). Thus, the information - if preserved - must remain within the black hole. This requires a larger set of possible interior states than the strong interpretation of the Bekenstein-Hawking entropy accounts for, as that shrinks during the evaporation process to $S=M_{\mathrm{f}}^{2}$ while the information content increases because it contains, in addition to the original state of the star that collapsed accross the horizon, the entangled partners of the Hawking radiation. This is the inevitable consequence from a conservative approach, and it applies while the black hole is still in the semi-classical regime 4 . The conclusion must be that the strong form of the Bekenstein-Hawking entropy is false and a weaker form is correct. The only candidate we are aware of for a weaker form of black hole entropy is that given above.

We further note that the strong form of the Bekenstein bound is explicitly violated by highly entropic states, considered in [30] and constructed in [31]. There is in General Relativity no obstacle in principle to solutions with arbitrarily small ADM mass and arbitrarily large entropy, and we have seen that they are a necessary consequence of Hawking evaporation without modifications in the semi-classical regime. The presence of such objects does not require any radical assumptions, whereas their avoidance does. It does thus seems puzzling why this option has not received much more attention as a possible solution to the black hole information loss problem. One objection against their existence is that they lead to singularities, but if singularities are removed by quantum dynamics then this cannot be an objection. Another objection is that they are in conflict with the strong form of the Bekenstein entropy bound. But this conflict can be resolved by accepting the weak form of the Bekenstein-Hawking entropy 5 .

We next turn to the other often raised objection against stable or quasi-stable remnants.

\footnotetext{
${ }^{4} \mathrm{~A}$ similar argument is the 'Hawking radiation cycle' discussed in [46].

${ }^{5}$ Another way to say this is that if one believes in the strong form of the Bekenstein-Hawking entropy and so has a problem with remnants, one has actually has a more serious problem.
} 


\section{Remnants must have an infinite cross section for pair production}

This argument begins with the assertion that effective field theory applies to remnants, so that they should, for purposes of physics above an length scale $\lambda_{c} \gg l_{\mathrm{Pl}}$, be treated as point particles which can be created and annihilated and described by local field operators [23, 24]. This is asserted to be the case because their physical diameter is much smaller than $\lambda_{c}$. However they must come in an infinite numbers of varieties because they have to contain the quantum information needed to restore the pure state of a thermal radiation produced by a potentially arbitrarily large initial black hole mass, as discussed above. Since the couplings in effective field theory can not resolve any structure with a scale much below $\lambda_{c}$, each of these species must have the same pair production cross-section. Even if the pair production cross section for one of these species is exponentially suppressed, it is multiplied by an infinite factor, so that if they existed there would be an infinite pair production rate for remnants in arbitrarily weak background fields, as long as the available energy exceeds two times the remnant mass.

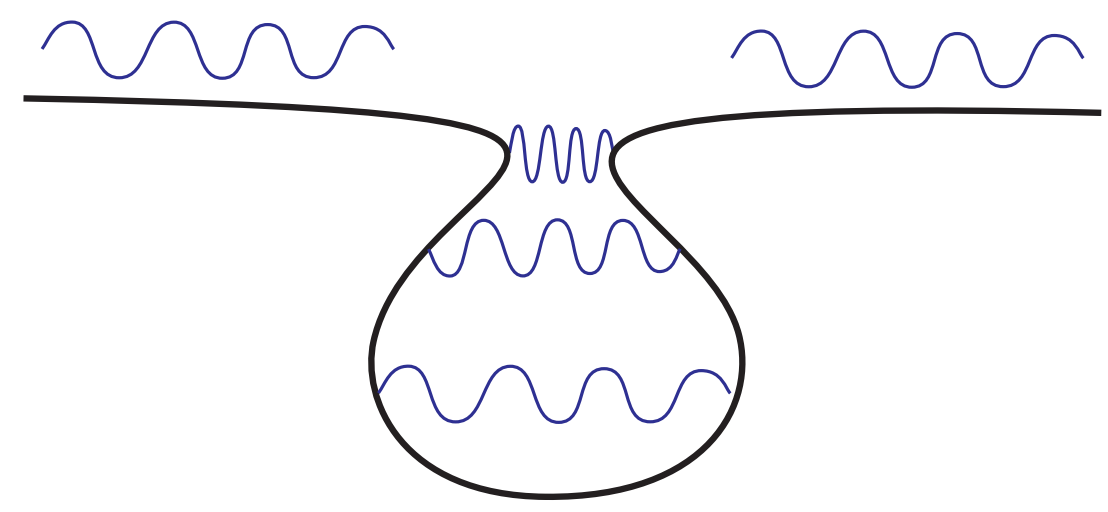

Figure 7: Schematic picture of a "bag of gold", ie a remnant with a large internal volume, embedded in an asymptotically flat space. Shown is a mode $|k\rangle$ which is long wavelength both far from and within the bag of gold to illustrate why it is implausible in an effective field theory long wavelength modes outside the remnant decouple from degrees of freedom within the remnant.

The problem with this argument is twofold.

First, it must be possible to define an effective field theory in this context. This depends on making a separation between modes with wavelength shorter and longer than $\lambda_{c}$, and integrating over the former to find an effective theory for the latter. However note that a characteristic property of a black hole is the blueshift of frequencies when one approaches the horizon. This represents a position dependent change for wavelengths that is specific to strongly curved backgrounds and is not a feature of flat space quantum field theory. There is then no distinction between short and long wavelength modes that is valid in the whole spacetime.

Related to this is our second point. Remnants are end stages of black hole evaporation, they are neither point particles nor are they in the semi-classical regime. They may be very small on the outside, because they can be entirely contained within a small surface 
area. But their interior can be a throat that opens up into an arbitrarily large internal volume. As has been discussed in the literature, the interior of the remnant is a region that can have a volume large compared to $M_{\mathrm{f}}^{3}$ and indeed be growing. This is the "bag of gold" scenario, discussed in [32], and illustrated in Figure 7. In this case, the excited modes of fields corresponding to whatever formed the black hole, whatever fell into it, and the unfortunate partner of each quanta of Hawking radiation, all end up here and as the interior expands will have very subplanckian wavelengths.

An effective field theory approach to remnants would only be possible to the extent that long wavelength modes with $\lambda>\lambda_{c} \gg l_{\mathrm{Pl}}$ decouple from the degrees of freedom in the interior of the remnant, which can distinguish the different possible internal states of the remnant. However, it is doubtful this is the case. Consider a mode such as that shown in Figure 7 which is very sub-Planckian in a region of space-time far from, or prior to, the formation of the black hole, very high frequency near the apparent horizon, but again very long wavelength in the interior of the horizon. Note that as it approaches the apparent horizon the mode $|k\rangle$ is very blue shifted, and it continues to blue shift as it comes closer to the region where the classical singularity would have been. After it enters the expanding bag of gold region it can red shift again. If the bag of gold is expanding the mode in the interior can be redshifted again to arbitrarily long wavelength.

It is thus a long wavelength mode in the exterior region that can interact strongly with the degrees of freedom inside the remnant. Its existence precludes the application of effective field theory to remnants. Moreover, the existence of such modes makes it impossible to assume that the amplitude for interaction between a long wavelength external mode and a remnant is independent of the degrees of freedom in the interior of the remnant. But this is a key assumption in the argument we sketched above for an infinite pair production rate for remnant:6. One might argue that in the presence of a horizon the interior is disconnected from the exterior. However, in a quantum theory, these regions are not disconnected as long as Hawking radiation, which can be understood as a tunneling process, is possible. A stable remnant does by assumption no longer emit Hawking radiation, but whenever something falls in its mass increases and it will emit until it has reached the remnant mass again.

There is in fact no more reason to think that a black hole remnant should be created by a local operator, than any other region of spacetime with an arbitrary large volume and high entropy. The geometry of the bag of gold to the interior of a remnant throat could be as large, weakly curved and have as many degrees of freedom and at least as much entropy as a star. Indeed, we would expect many black hole remnants to be the future evolutions of stars.

The situation is unchanged if the black hole is produced by quantum tunneling, rather than by classical gravitational collapse. So long as the remnant does not completely pinch off, there is an event horizon that an external quanta's wavefunction may fall into. Thus, the case for the essential assumption needed to set up a description of a remnant by a single local operator in effective field theory, which is the decoupling of its internal degrees

\footnotetext{
${ }^{6}$ Related to this, one can argue that the interaction between any long wavelength mode and a highly entropic internal state should be suppressed the higher the entropy of the internal state.
} 
of freedom from long wave length degrees of freedom of the exterior, is not conclusive. If an effective description of such remnants is possible at all, their couplings should depend on their internal state even in the low energy limit. This possibility has been discussed for some while [33, 34, 35, 24, 23, 36] in the mid 90s but no consensus appears to have been attained about it 7 .

Other objections against baby universes have been addressed in [37].

\section{Life time of decaying remnants}

If there is not a permanent remnant, then there arises the question how long the last stages of black hole evaporation will last. The Planck scale remains of the black hole may slowly decay, potentially exceeding the lifetime of the universe, or it may quickly dissolve on microscopic timescales. We will here not advocate a specific answer, but just mention several points of view.

A standard objection to the fast decay is that there is a large amount of quantum information, proportional to the entropy of the Hawking radiation, $\sim M_{\mathrm{i}}^{2} / m_{\mathrm{Pl}}^{2}$, where $M_{\mathrm{i}}$ is the initial mass of the collapsing matter, in an object of eventually very ADM small mass $\sim m_{\mathrm{Pl}}$. At one quanta per bit, each quanta will have a tiny amount of energy, $\sim m_{\mathrm{Pl}}^{3} / M_{\mathrm{i}}^{2}$ and the more information, the less energy per bit one has. The emission of such a small amount of energy takes a long time, and since the bits of information come out of a very small region of spacetime they can not be emitted together because their wave-functions would overlap and spoil the encoding of information.

The only actual calculations for the resulting lifetime that we are aware of are 38 and [39]. Preskill [15] offers an heuristic argument based on the former references, and Giddings [40] presents a similar estimate. Reference [38] however make use of the small volume of the remnant and [39] uses a moving mirror analogy that presupposes the final quantum gravitational object has still an horizon, neither of which might be applicable to the actual endstate.

If one considers the recovery of information takes place only in the Planckian phase, as the most conservative approach would indicate, this requires that the strong form of black hole entropy be false, as we discussed above. If one insists instead on the strong form then the black hole must leak information correlated with the thermal Hawking radiation, as the black hole evaporates, so that at any time, $t$, during the evaporation, no more than $\sim \exp \left(M(t)^{2}\right)$ qbits are needed to restore the external state to a pure state. On this assumption there is very little information in the black hole by the time it evaporates down to the Planck mass and the remains can quickly dissolve. However, the emission of information in the Hawking radiation typically involves non-local physics in areas where quantum gravitational effects are expected to be negligible to get the information from the quantum gravitational region to and/or through the horizon.

The scenario with leakage prior to the Planckian phase does seem to be indicated by the calculations of Ashtekar et al [10]. It is also supported by considerations of quantum information theory as argued by Hayden and Preskill [41] - although we note their argu-

\footnotetext{
${ }^{7}$ We note that the inconclusiveness of these investigations has been pointed out already eg in [14, 29].
} 
ment assumes the strong form of black hole entropy meaning it assumes information can not stay inside and has to come out.

Taken together we see again that it is our lacking understanding of the strong quantum gravitational effects near the singularity that prohibit us to formulate a satisfactory solution to the information loss problem, and more investigation is required to understand the properties of Planck-size, Planck-mass objects.

\subsection{AdS/CFT}

Let us discuss the implications of the AdS/CFT conjecture from the point of view above. To do this we have to state a couple of standard modifications of our earlier definitions.

A partly semi-classical quantum spacetime $\mathcal{Q S T}$ is asymptotically AdS if its classical approximation $\left(\mathcal{R},\left\langle g_{a b}\right\rangle\right)$ is asymptotically AdS.

We note that the boundary of an asymptotically AdS spacetime is a timelike surface $\mathcal{S}$, of one dimension lower than the AdS space, on which there is a flat Minkowski metric. One can then define an asymptotic time coordinate, $t$, both in the asymptotically AdS metric and the Minkowski spacetime.

An horizon of an asymptotically AdS quantum spacetime $\mathcal{Q S T}$ is the boundary of the causal past of $\mathcal{S}$ of its classical approximation $\left(\mathcal{R},\left\langle g_{a b}\right\rangle\right)$.

The AdS/CFT conjecture is then the following [42].

AdS/CFT conjecture: There is a quantum theory of gravity $\mathcal{Q G}$ which encompasses partly semi-classical asymptotically AdS spacetimes. It has a Hilbert space $\mathcal{H}_{\text {bulk }}$ and a Hamiltonian $H_{\text {bulk }}$ which generates time evolution in the asymptotic time coordinate $t$. There is also a conformal field theory $\mathcal{Q C}$ on $\mathcal{S}$ with a Hilbert space $\mathcal{H}_{\text {boundary }}$ and Hamiltonian $H_{\text {boundary }}$ that generates time evolution in the same $t$. There is also another Hamiltonian in $\mathcal{H}_{\text {boundary }}$, given by $H_{\mathrm{CFT}} \propto H_{\text {boundary }}+\mathcal{D}$ where $\mathcal{D}$ is another generator of the conformal group acting on $\mathcal{S}$.

These two theories are isomorphic to one another in the sense that there exists an isomorphism $\mathcal{I}_{\mathrm{AdS} / \mathrm{CFT}}$

$$
\mathcal{I}_{\text {AdS } / \mathrm{CFT}}(t): \mathcal{H}_{\text {bulk }} \leftrightarrow \mathcal{H}_{\text {boundary }}
$$

that takes the operator algebras to each other.

$$
\mathcal{I}_{\mathrm{AdS} / \mathrm{CFT}}(t): \mathcal{A}_{\mathrm{bulk}} \leftrightarrow \mathcal{A}_{\mathrm{CFT}}
$$

and in particular

$$
\mathcal{I}_{\text {AdS } / \mathrm{CFT}}(t): H_{\text {bulk }} \leftrightarrow H_{\mathrm{CFT}}
$$

such that time evolution commutes with $\mathcal{I}_{\text {AdS/CFT }}(t)$ in the sense that

$$
\mathcal{I}_{\mathrm{AdS} / \mathrm{CFT}}(t) \cdot e^{-\imath H_{\mathrm{CFT}} t}=e^{-\imath H_{\mathrm{bulk}} t} \cdot \mathcal{I}_{\mathrm{AdS} / \mathrm{CFT}}(t) .
$$


It follows from the statement of this conjecture that the $\mathcal{Q G}$ should be quantum nonsingular. Given a complete spacelike surface $\Sigma$ in a semiclassial region of the bulk, there should be an invertible inclusion map, $\mathbf{I}_{\Sigma}$, of the operator algebras, or density matrices such that pure states of the full theory restrict to pure states of the linearized theory.

$$
\begin{array}{lll}
\mathbf{I}_{\Sigma}: & \mathcal{A}_{\text {bulk }} \rightarrow \mathcal{A}_{\Sigma} \\
\mathbf{I}_{\Sigma}: & \mathcal{H}_{\text {bulk }} \rightarrow \mathcal{H}_{\Sigma}
\end{array}
$$

Now consider two such complete spacelike slices, $\Sigma_{1}$ and $\Sigma_{2}$, which intersect $\mathcal{S}$ at boundary times, $t_{1}$ and $t_{2}$. Given a state $\left|\Psi_{\text {boundary }}, t_{1}\right\rangle$ in $\mathcal{H}_{\text {boundary }}$, we can map it with $\mathcal{I}_{\text {AdS } / \text { CFT }}^{-1}$ to get a state $\left|\Psi_{\text {bulk }}, t_{1}\right\rangle$ in $\mathcal{H}_{\text {bulk }}$. We can then use $\mathbf{I}_{\Sigma_{1}}$ to map this to a state in the Hilbert space of the linearized theory on $\Sigma_{1}$, which is $\mathcal{H}_{\Sigma_{1}}$.

We can also use $e^{-\imath H_{\mathrm{CFT}}\left(t_{2}-t_{1}\right)}$ to evolve $\left|\Psi_{\text {boundary }}, t_{1}\right\rangle$ to a state $\left|\Psi_{\text {boundary }}, t_{2}\right\rangle$ in $\mathcal{H}_{\text {boundary }}$ and then again use $\mathcal{I}_{\text {AdS } / \text { CFT }}^{-1}$ to map it to a pure state $\left|\Psi_{\text {bulk }}, t_{2}\right\rangle$ in $\mathcal{H}_{\text {bulk }}$. We then use $\mathbf{I}_{\Sigma_{2}}$ to map this to a state in the Hilbert space of the linearized theory on $\Sigma_{2}$, which is $\mathcal{H}_{\Sigma_{2}}$.

Since $e^{-\imath H_{\mathrm{CFT}}\left(t_{2}-t_{1}\right)}$ is unitary and $\mathcal{I}_{\mathrm{AdS} / \mathrm{CFT}}^{-1}$ is an isomorphism this gives a unitary map on from $\mathcal{H}_{\Sigma_{1}}$ to $\mathcal{H}_{\Sigma_{2}}$. Thus, if the AdS/CFT conjecture is true, the evolution in the bulk between any two such hypersurfaces is unitary and there can not be information loss. There can however also not be a quantum singularity between these slices.

Thus, if the assumptions of this argument are correct then we are left with scenarios three and four.

\subsection{Curvature singularities}

Given a theory of quantum gravity, one could consider alternative notions of singular spacetimes. A more natural definition than the one we have used here might be thought to be one in which a singularity occurs when expectation values of operators representing scalar invariants of the curvature tensor diverge. A quantum spacetime could then be said to be curvature non-singular if there is no event where the expectation value of a scalar function of the curvature tensor diverges. One then could ask whether both notions of singular space-times coincide. Given that we do not know the fundamental theory we can only make some general remarks about this.

For spacetime to be quantum singular, there can be no two initial states that in the quantum spacetime evolve into one and the same, and such arrive as the same state on the final hypersurface. For this to happen, it is however sufficient if there is an attractor for states that erases their differences. Without further knowledge about the relation between the spacetime and the quantum fields propagating in it, it is impossible to say whether this attractor must be accompanied by a divergence.

Similarly, whether or not a curvature singularity implies spacetime is quantum singular for the quantum field depends on the evolution laws coupling the spacetime to the propagating field. One could then have three different cases in which a curvature singularity would not imply a quantum singularity for the propagating quantum field: 
1. Even though space-time has a curvature singularity, the quantum field has not, e.g. because the quantum field does not resolve the structure, or gravity decouples in the high density limit.

2. Even though space-time has a curvature singularity no information runs into it, e.g. because it is redistributed 8 and survives elsewhere. Since this means the information has to get out of the horizon before it is gone, this typically implies non-local effects.

3. The singularity itself can carry information and in such a way pass it on through the quantum region, this is realized e.g. in the proposal by Horowitz and Maldacena [43.

\section{Discussion}

We would now like to make some comments on the debate on the information loss problem, in light of the conclusions we have reached.

What seems to us puzzling about much of the discussion in the literature is that the obvious conservative solution, that the singularity is removed and unitarity so restored, has not been given more attention. The information loss problem seems paradoxical only if one believes it can not simply be blamed on our lacking understanding of the processes in the quantum gravitational region. This is the case only if one accepts as true the arguments that have been raised against the conservative solutions we have classified here, arguments which we do not believe are definitive, precisely because we lack understanding about the quantum gravitational phase. If one believes these arguments however, then one has reason to construct more fanciful and radical solutions.

Our main conclusion is that there is only need to invent radical solutions in the case that the right quantum theory of gravity will not eliminate the singularity in Figures 1 and 2. Were this the case, then we would apparently have to solve problem of how to evolve unitarily between $\mathcal{I}^{-}$and $\mathcal{I}^{+}$in Figures 2 . This may lead us to radical mechanisms such as black hole complementarity that imply non-local transfers of large amounts of quantum information over large space-like intervals [3], or to argue that a non-perturbative completion of gravity displays non-locality on the scale of the horizon [44]. But that problem is moot if the actual causal structure of a quantum black hole spacetime looks like Figure 3 or 4 .

We then find it encouraging that one of the most detailed quantum gravity calculation of the fate of an evaporating black hole that so far has been done shows that the singularity is restored and unitarity evolution between $\mathcal{I}^{-}$and $\mathcal{I}^{+}$is confirmed [10]. This work shows that, at least in the example studied, once the singularity is eliminated, the causal structure of a black hole that forms from gravitational collapse and then evaporates is that of Minkowski spacetime, so that restoration of unitary evolution is almost trivial.

Assuming the singularity is eliminated, there remains the question of whether the information contained in the initial state returns to infinity, as in option 3 or remains

\footnotetext{
${ }^{8}$ We assume that the no-cloning theorem holds and exclude that information can be copied.
} 
trapped in a baby universe or permanent remnant as in option 4 . In the case of option 3 (which seems to be suggested by the results in [10]), it is not enough to invoke unitarity, one has still to understand how the information comes out. So, not surprisingly, more work is needed concerning the Planck scale regime, when the semiclassical approximation breaks down, but this is no reason to give up and retreat to radicalism. If one takes the conservative point of view that we have presented here, then an important issue is to understand the properties of the highly entropic endstate of the black hole evaporation. We thus hope to encourage more investigation of this subject.

\section{Conclusions}

We have argued here that elimination of the singularity is sufficient to resolve the quantum information problem raised by Hawking in the context of black hole evaporation. We reached this conclusion by noting that, in the absence of knowing the theory of quantum gravity, an appropriate condition for a quantum spacetime to be non-singular is the existence of reversible maps between Hilbert spaces describing the linearized quantum fluctuations of quantum fields between spacelike surfaces. This then provides a useful definition of non-singularity to classify the options for resolving the information paradox without invoking departures from the semi-classical description outside the region where quantum gravitational corrections can be expected to be strong.

As we have seen, in the cases where the singularity is eliminated, no information loss can occur. The scenarios which resolve it are either that the black hole evaporates completely, or leaves a permanent massive remnant, or forms a baby universe. In the first case there is no real horizon, but in the latter cases there is. Which is the actual resolution depends on the details of the quantum theory of gravity. Moreover, there is no reason in principle why a single theory could not have states where each of these options are realized, following the generic expectation that in a quantum theory everything that can happen will happen unless explicitly forbidden. We further have seen that the arguments so far offered against short- and long-lived remnants or baby universes are not convincing due to a lacking investigation of the properties of highly entropic objects with curvature in the Planckian regime.

Finally, we considered the issue in the context of the AdS/CFT correspondence and reached the conclusion that the conjecture of equivalence of the bulk and boundary theory requires that the bulk quantum spacetime be non-singular, in the sense defined here.

\section{Acknowledgements}

We are grateful to Abhay Ashtekar, Steve Giddings, Stephen Hsu, Ted Jacobson and Don Page for conversations and correspondence on this issue. Research at Perimeter Institute for Theoretical Physics is supported in part by the Government of Canada through NSERC and by the Province of Ontario through MRI. 


\section{References}

[1] S. W. Hawking, Black hole explosions?, Nature. 248 (5443): 3031 (1974).

[2] S. W. Hawking, "Breakdown Of Predictability In Gravitational Collapse," Phys. Rev. D 14, 2460 (1976).

[3] L. Susskind, L. Thorlacius, J. Uglum, The Stretched Horizon and Black Hole Complementarity, arXiv:hep-th/9306069v2, Phys.Rev. D48 (1993) 3743-3761; David A. Lowe, Joseph Polchinski, Leonard Susskind, Larus Thorlacius, John Uglum, Black Hole Complementarity vs. Locality, , arXiv:hep-th/9506138v1, Phys.Rev. D52 (1995) 6997-7010.

[4] S. B. Giddings, D. J. Gross and A. Maharana, Gravitational effects in ultrahighenergy string scattering, Phys. Rev. D 77, 046001 (2008) arXiv:0705.1816 [hep-th]]; S. B. Giddings, Locality in quantum gravity and string theory, Phys. Rev. D 74, 106006 (2006) arXiv:hep-th/0604072.

[5] J. W. York, Dynamical Origin Of Black Hole Radiance, Phys. Rev. D 28, 2929 (1983).

[6] V. P. Frolov, M. A. Markov and V. F. Mukhanov, Black Holes as Possible Sources of Closed and Semiclosed Worlds, Phys. Rev. D 41, 383 (1990).

[7] V. P. Frolov, M. A. Markov and V. F. Mukhanov, Through a Black Hole into a new Universe?, Phys. Lett. B 216, 272 (1989).

[8] L. Modesto, Loop quantum gravity and black hole singularity, arXiv:hep-th/0701239 ; Gravitational collapse in loop quantum gravity, arXiv:gr-qc/0610074, Int.J.Theor.Phys.47:357-373,2008 .

[9] C. G. . Callan, S. B. Giddings, J. A. Harvey and A. Strominger, Evanescent black holes, Phys. Rev. D 45, 1005 (1992) arXiv:hep-th/9111056.

[10] A. Ashtekar, V. Taveras and M. Varadarajan, Information is Not Lost in the Evaporation of 2-dimensional Black Holes, Phys. Rev. Lett. 100, 211302 (2008) arXiv:0801.1811 [gr-qc]].

[11] V. P. Frolov and G. A. Vilkovisky, Phys. Lett. B 106, 307 (1981).

[12] Abhay Ashtekar, Martin Bojowald, Quantum geometry and the Schwarzschild singularity, arXiv:gr-qc/0509075, Class.Quant.Grav. 23 (2006) 391-411; Black hole evaporation: A paradigm, arXiv:gr-qc/0504029, Class.Quant.Grav. 22 (2005) 3349-3362.

[13] Sean A. Hayward, The disinformation problem for black holes, arXiv:gr-qc/0504037; C.R. Stephens, G. 't Hooft, B.F. Whiting, Black Hole Evaporation without Information Loss, arXiv:gr-qc/9310006, Class.Quant.Grav. 11 (1994) 621-648

[14] D. N. Page, Black hole information, arXiv:hep-th/9305040. 
[15] J. Preskill, Do black holes destroy information?, arXiv:hep-th/9209058.

[16] Hawking and Ellis, The large scale structure of space time, Cambridge University Press (1975).

[17] N. D. Birrell and P. C. W. Davies, Quantum Fields in Curved Space, Cambridge University Press (1984).

[18] L. H. Ford, Quantum field theory in curved spacetime, arXiv:gr-qc/9707062.

[19] B. DeWitt, Dynamical Theory of Groups and Fields, Gordon and Breach (1965).

[20] S. W. Hawking, Particle Creation by Black Holes, Comm. Math. Phys. 43, 199-220 (1975).

[21] S. B. Giddings, Quantum mechanics of black holes, 'arXiv:hep-th/9412138.

[22] A. Strominger, Les Houches lectures on black holes, arXiv:hep-th/9501071.

[23] S. B. Giddings, Comments On Information Loss And Remnants, Phys. Rev. D 49, 4078 (1994) arXiv:hep-th/9310101.

[24] S. B. Giddings, Constraints on black hole remnants, Phys. Rev. D 49, 947 (1994) arXiv:hep-th/9304027.

[25] L. Susskind, Trouble For Remnants, arXiv:hep-th/9501106.

[26] F. Markopoulou, L. Smolin, Holography in a quantum spacetime, arXiv:hep-th/9910146.

[27] L. Smolin, The strong and weak holographic principles, arXiv:hep-th/0003056, Nucl.Phys. B601 (2001) 209-247

[28] R. D. Sorkin, The statistical mechanics of black hole thermodynamics, arXiv:gr-qc/9705006.

[29] T. Jacobson, On the nature of black hole entropy, arXiv:gr-qc/9908031.

[30] D. Marolf and R. D. Sorkin, On the status of highly entropic objects, Phys. Rev. D 69, 024014 (2004) arXiv:hep-th/0309218.

[31] S. D. H. Hsu and D. Reeb, Black hole entropy, curved space and monsters, Phys. Lett. B 658, 244 (2008) [arXiv:0706.3239 [hep-th]].

[32] J.A. Wheeler, in Relativity, Groups and Topology, edited by B DeWitt and C DeWitt, p 408-31 Gordon and Breach (1974)

[33] T. Banks and M. O'Loughlin, Classical and quantum production of cornucopions at energies below $10^{1} 8 \mathrm{GeV}$, Phys. Rev. D 47, 540 (1993) [arXiv:hep-th/9206055]. 
[34] T. Banks, M. O'Loughlin and A. Strominger, Black hole remnants and the information puzzle, Phys. Rev. D 47, 4476 (1993) |arXiv:hep-th/9211030|.

[35] T. Banks, Lectures on black holes and information loss, Nucl. Phys. Proc. Suppl. 41, 21 (1995) arXiv:hep-th/9412131.

[36] S. B. Giddings, Why Aren't Black Holes Infinitely Produced?, Phys. Rev. D 51, 6860 (1995) arXiv:hep-th/9412159.

[37] S. D. H. Hsu, Spacetime topology change and black hole information, Phys. Lett. B 644, 67 (2007) arXiv:hep-th/0608175.

[38] Y. Aharonov, A. Casher and S. Nussinov, The Unitarity Puzzle And Planck Mass Stable Particles, Phys. Lett. B 191, 51 (1987).

[39] R. D. Carlitz and R. S. Willey, The Lifetime of a Black Hole, Phys. Rev. D 36, 2336 (1987).

[40] S. B. Giddings, Black holes and massive remnants, Phys. Rev. D 46, 1347 (1992) arXiv:hep-th/9203059.

[41] P. Hayden and J. Preskill, Black holes as mirrors: quantum information in random subsystems, JHEP 0709, 120 (2007) [arXiv:0708.4025 [hep-th]].

[42] J. Maldacena, The large $N$ limit of superconformal field theories and supergravity, Adv. Theor. Math. Phys. 2 (1998) 231 [hep-th/9711200]; E.Witten, Anti-de Sitter space and holography, Adv. Theor.Math. Phys. 2 (1998) 253 hep-th/9802150]; S. S. Gubser, I. R. Klebanov and A. M. Polyakov, Gauge theory correlators from noncritical string theory, Phys. Lett. B 428 (1998) 105 |hep-th/9802109|.

[43] G. T. Horowitz and J. M. Maldacena, The black hole final state, JHEP 0402, 008 (2004) arXiv:hep-th/0310281.

[44] S. B. Giddings, Quantization in black hole backgrounds, Phys. Rev. D 76, 064027 (2007) |arXiv:hep-th/0703116]; S. B. Giddings, (Non)perturbative gravity, nonlocality, and nice slices, Phys. Rev. D 74, 106009 (2006) arXiv:hep-th/0606146.

[45] R. M. Wald, The Thermodynamics of Black Holes, Living Rev. Rel. 4, 6 (2001).

[46] D. Marolf, Black Holes, AdS, and CFTs, arXiv:0810.4886 [gr-qc].

[47] B. Hasslacher and E. Mottola, Asymptotically Free Quantum Gravity And Black Holes, Phys. Lett. B 99, 221 (1981). 